\title{
Prevalence of ECG abnormalities among adults with metabolic syndrome in a Nigerian Teaching Hospital
}

\author{
Adeoye Abiodun ${ }^{1}$, Adebayo Oladimeji ${ }^{2}$, Tayo Bamidele ${ }^{3}$, Adebiyi Adewole ${ }^{4}$, Owolabi Mayowa ${ }^{5}$
}

1. Department of Medicine, College of Medicine,University of Ibadan.

2. University College Hospital, Medicine.

3.Loyola University, Population Health.

4. University College Hospital and College of Medicine, Department of Medicine.

5. College of Medicine, University of Ibadan, Ibadan, Neurology Unit, Department of Medicine.

\begin{abstract}
Background: Co-existence of metabolic syndrome (MetS) and electrocardiography (ECG) abnormalities heightens the risk of sudden cardiac death. However, there is a gap in evidence of how ECG changes cluster among continental Africans with or without MetS.

Methods: We included 491 participants with interpretable ECG tracings who were consecutively recruited into the Cardiovascular Risk Prediction Registry (CRP). CRP is a registry of newly presenting patients into cardiology clinic of the University College Hospital, Nigeria, with a main objective of cardiovascular risk stratification to prevent cardiovascular morbidity and mortality. Using the International Diabetic Federation (IDF) criteria they were divided into those with metabolic syndrome and non-metabolic syndrome.

Results: Four hundred and ninety-one participants comprising $48.3 \%$ women with mean age $53.72 \pm 15.2$ years who met the IDF criteria with complete ECG interpretations were analyzed with $44.2 \%$ (men 38.6\%; women $50.2 \%$ ) of the participants having MetS while 74\% had ECG abnormalities. Compared to women, men had higher mean serum total cholesterol, creatinine, smoking, and alcohol use, family history of hypertension and diabetes mellitus, QT prolongation, LVH plus or minus strain pattern, and ECG abnormalities in general. Women were heavier, had higher heart rate and proportions of MetS. ECG findings among those with or without MetS were not significantly different. In men, IDF metabolic score was associated with conduction abnormalities $(p=0.039)$ and combined ECG abnormality $(p=0.042)$ which became more significant with an exclusion of QT prolongation $(\mathrm{p}=0.004)$. Also, IDF abdominal obesity was associated with QT prolongation $(\mathrm{p}=0.017)$, combined ECG abnormality $(\mathrm{p}=0.034)$ while HDLc correlated with ECG abnormalities $(0.037)$ in men. There was no significant associations of components of metabolic syndrome with ECG abnormalities among women.

Conclusion: There was a high prevalence of MetS and abnormal ECG among the studied population. Abnormal ECG findings were more common in men with no differential association in people with or without MetS. However, a significant association existed between certain components of MetS and ECG abnormalities in men only. Male gender and HDLc were independent predictors of ECG Abnormalities.
\end{abstract}

Keyword: Electrocardiography, metabolic syndrome, africans.

DOI: https://dx.doi.org/10.4314/ahs.v19i4.4

Cite as: Abiodun A, Oladimeji A, Bamidele T, Adewole A, Mayowa O. Prevalence of ECG abnormalities among adults with metabolic syndrome in a Nigerian Teaching Hospital. Afri Health Sci. 2019;19(4):2829-2838. bttps:// dx.doi.org/10.4314/abs.v19i4.4

\section{Corresponding author: \\ Adeoye Abiodun, \\ Department of Medicine, \\ College of Medicine, \\ University of Ibadan. \\ Email: adeoyemoshood@yahoo.com}

\section{Introduction}

Metabolic syndrome (MetS) is a constellation of cardio-metabolic risk factors that contribute immensely to the burden of cardiovascular morbidity and mortality worldwide. The prevalence of MetS varies depending on the diagnostic criteria and study populations ${ }^{1-3}$. Due to epidemiologic transitions arising mainly from an unhealthy lifestyle, the incidence of MetS is on a steady rise

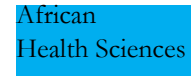

(C) 2019 Abiodun et al. Licensee African Health Sciences. This is an Open Access article distributed under the terms of the Creative commons Attribution License (https://creativecommons.org/licenses/BY/4.0), which permits unrestricted use, distribution, and reproduction in any medium, provided the original work is properly cited. 
in low and middle-income countries (LMICs), as well as high-income countries (HICs). Prevalence as much as $40 \%$ has been reported in some HICs, while in Nigeria it has been found to be about $30 \% 0^{4,5}$. This burden is expected to increase in near future with it attendant high risk of cardiovascular disabilities and sudden deaths $s^{6,7}$.

Metabolic syndrome has been associated with sudden cardiac death(SCD). ${ }^{8}$ In ARIC (Atherosclerosis Risk in Communities) Study, compared with participants without MetS, those with metabolic syndrome independent of race and ethnicity, had approximately $70 \%$ increased the risk of sudden cardiac death. Elevated blood pressure, impaired fasting glucose and low -density lipoprotein cholesterol as Mets components were shown to be the independent determinants of SCD. ${ }^{9}$

Interestingly, each of the components of MetS such as dyslipidemia, elevated blood pressure, insulin resistance \pm glucose intolerance, as well as abdominal obesity has been shown to cause cardiac structural damages and arrhythmias $^{10-13}$. Coexistence, therefore of MetS and ECG abnormalities may increase the risk of sudden cardiac death. However, there is a paucity of data on the association of cardiac arrhythmias and metabolic syndrome. ${ }^{14}$ Early detection of these cardiac abnormalities using baseline 12 -lead ECG which is simple, noninvasive and cost -effective can help identify at-risk individuals for prompt and focused management.

Studies differ on the prevalence and associations of components of MetS with ECG abnormalities. In certain populations, a high prevalence of ECG abnormalities in metabolic syndrome were reported ${ }^{10,15-18}$. While coronary artery disease and its attendant ECG changes have similar findings among Caucasians, and same are rarely reported among Africans ${ }^{19,20}$. Despite the huge burden of stroke, heart failure and kidney failure in sub-Sahara Africa (SSA), data on ECG changes among metabolic syndrome patients alone are lacking. In order to address this gap and to show how ECG changes cluster among Africans with MetS, we report ECG changes in a Nigerian population with MetS compared to those without MetS.

\section{Methods}

\section{Subjects and methods}

This cross-sectional and comparative study was conducted over a period of one year from July 2014 to June 2015 following approval by the Joint University of Ibadan (UI)/ University College Hospital (UCH) Ethics Committee approval reference number UI/EC/14/0136 dated 19 ${ }^{\text {th }}$ June 2014. Four hundred and ninety-one adult patients with complete data for the diagnosis of metabolic syndrome and interpretable ECG tracings from CRP registry were studied. Cardiovascular Risk Prediction (CRP) is a registry of newly presenting patients into the cardiology division, medical out-patient department of University College Hospital Ibadan. The main aim of the registry is to risk stratify the patients at entry using Framingham and QRISK2 cardiovascular risk score for prompt management of at-risk individuals to prevent future cardiovascular morbidity and mortality.

Biodata of the participants was obtained using pretested questionnaires containing information on demographics, family history of cardiovascular diseases, life-styles and medication history. The contact information of the participants and their next of kin were obtained for communication during follow-up.

\section{Assessment of anthropometric measurement}

Anthropometric measurements including height, weight, waist and arm circumferences were obtained by trained nurses at the medical outpatient clinic. Height was measured without shoes to the nearest centimeter using a ruler attached to the wall. Weight was measured to the nearest $0.1 \mathrm{~kg}$ on an electronic scale, with the subject wearing light outdoor clothing. Waist circumference was measured at the end of expiration at a mid-way between the rib cage and the iliac crest using an anthropometric measuring tape. Average of three measured waist circumferences, recorded to the nearest tenth of a centimeter was obtained for analysis.

Obesity was classified based on BMI in $\mathrm{kg} / \mathrm{m}^{2}$ as normal $(>20$ and $<25)$, overweight $(>25$ and $<30)$, obesity $(>30$ and $<35)$ and severe obesity $(\geq 35) 21$. Abdominal obesity was defined based on the International Diabetic Federation (IDF) criteria using waist circumference $(\geq 94 \mathrm{~cm}$ (men), $\geq 80 \mathrm{~cm}$ (female). Participants were further categorized as normal risk (men $<94 \mathrm{~cm}$; women $<80 \mathrm{~cm}$ ), increased risk (men $94-102 \mathrm{~cm}$; women $80-88$ $\mathrm{cm}$ ), or substantially increased risk (men $>102 \mathrm{~cm}$; women $>88 \mathrm{~cm}$ ) on the basis of the World Health Organization's standards for increased health risk associated with waist circumference $e^{22,23}$. 


\section{Assessment of blood pressure}

The clinic blood pressure was measured in a relaxed sitting position using a standard Omron (HEM711DLX) with blood pressure apparatus on the left arm, placed at heart level after 5-minute rest and using a cuff of appropriate size with legs uncrossed. Three BP measurements were obtained with a minimum interval of one minute and average of the last two measurements was used in the present analysis. Hypertension was defined as systolic blood pressure $(\mathrm{SBP}) \geq 140 \mathrm{mmHg}$ and/or diastolic blood pressure $(\mathrm{DBP}) \geq 90 \mathrm{mmHg}$ or being on antihypertensive treatment.

\section{Electrocardiography}

All participants for this study had resting 12-lead ECG, using a commercially available CONTEC® Workstation Model CONTEC EC8000G, ECG machine (Made in China) at speed of $25 \mathrm{~mm} / \mathrm{s}$ and $1 \mathrm{mV} / \mathrm{cm}$ calibration. The entire ECG tracing used were inspected visually by the blinded ECG technicians in our non-invasive cardiology laboratory to detect technical errors, unrecorded leads and inadequate quality of tracings. Defective ones are repeated before data was extracted for use in the study. The ECG tracings were independently analyzed by the cardiologists who were unaware of the details of the clinical status of the patients. Abnormalities obtained from the ECGs were defined according to standard criteria24,25 (table 1). Left ventricular hypertrophy was diagnosed using the following criteria: Sokolow-Lyon voltage (sum of the amplitudes of $\mathrm{S}$ wave in $\mathrm{V} 1$ and $\mathrm{R}$ wave in $\mathrm{V} 5$ or $\mathrm{V} 6 \geq 3.5 \mathrm{mV}$ ). Repolarization abnormalities in leads V5 and/or V6 indicated typical strain when there was down-sloping convex ST segment with an inverted asymmetrical T-wave opposite to the QRS axis26,27. QT interval was determined using the tangent method28.

The measured QT interval was corrected for heart rate using the Bazett's formula. Prolonged QT interval was considered present when the QTc was $>450$ milliseconds and $>440$ milliseconds in women and men respectively. Presence of other ST-T changes was documented according to standard criteria ${ }^{24}$.

Table 1: Definitions of ECG Variables

\begin{tabular}{|c|c|}
\hline ECG Variables & Definitions \\
\hline Atrial fibrillation(Afib) & $\begin{array}{l}\text { Coarse fibrillatory waves, irregular QRS, rate } 400- \\
\text { 500beats per minutes }\end{array}$ \\
\hline Atrial flutter(Afl) & $\begin{array}{l}\text { Rate between } 250 \text { to } 350 \text { beats per minute, saw tooth } \\
\text { pattern P waves }\end{array}$ \\
\hline Sinus arrhythmia & $\begin{array}{l}\text { Variation of the P-P interval, from one beat to the next, of } \\
\text { at least } 0.12 \text { seconds, or } 120 \text { milliseconds }\end{array}$ \\
\hline $\begin{array}{l}\text { Premature Ventricular } \\
\text { Contraction(PVC) }\end{array}$ & Abnormal QRS shape and $\mathrm{QRS}=\geq 120 \mathrm{~ms}$ \\
\hline $\begin{array}{l}\text { Premature Supra } \\
\text { ventricular } \\
\text { contraction(PSVC) }\end{array}$ & $\begin{array}{l}\text { Presence of sinus tachycardia, atrial tachycardia, atrial } \\
\text { fibrillation, AVNRT complexes, atrial flutter, multifocal } \\
\text { atrial tachycardia, accelerated junctional tachycardia }\end{array}$ \\
\hline $\begin{array}{l}\text { Left atrial } \\
\text { enlargement(LAE) }\end{array}$ & $\mathrm{P}$ wave in lead II is greater than $\geq 120 \mathrm{~ms}$ \\
\hline Bi-atrial enlargement(BAE) & $\begin{array}{l}\mathrm{P} \text { wave in lead II is greater than } 120 \mathrm{~ms} \text { and higher than } \\
\geq 2.5 \mathrm{~mm}\end{array}$ \\
\hline $\begin{array}{l}\text { Left Ventricular } \\
\text { hypertrophy(LVH)Sokolow- } \\
\text { Lyon }\end{array}$ & $\mathrm{S}_{\mathrm{V}-\mathrm{I}}+\mathrm{R}_{\mathrm{V}-5}$ or $\mathrm{R}_{\mathrm{V}-6}=\geq 35 \mathrm{~mm}$ (whether male or female) \\
\hline $\begin{array}{l}\text { Left Ventricular } \\
\text { hypertrophy with strain }\end{array}$ & $\begin{array}{l}\mathrm{S}_{\mathrm{V}-\mathrm{I}}+\mathrm{R}_{\mathrm{V}-5} \text { or } \mathrm{R}_{\mathrm{V}-6}=\geq 35 \mathrm{~mm} \text { (whether male or female) } \\
\text { with } \mathrm{T} \text { waves changes }\end{array}$ \\
\hline $\begin{array}{l}\text { Right Ventricular } \\
\text { hypertrophy(RVH) }\end{array}$ & $\begin{array}{l}\mathrm{R} / \mathrm{S} \text { ratio of greater than } 1 \text { in lead } \mathrm{V} 1 \text { in the absence of } \\
\text { other causes or if the } \mathrm{R} \text { wave in lead } \mathrm{V} 1 \text { is greater than } 7 \\
\text { millimeters tall }\end{array}$ \\
\hline P duration & $\leq 0.120$ seconds \\
\hline QRS duration & $0.80-0.120$ seconds \\
\hline PR interval & $0.12-0.20$ seconds \\
\hline QT Interval & 0.40 seconds( for males) and 0.44 seconds (for females) \\
\hline
\end{tabular}




\section{Definition of metabolic syndrome}

Metabolic syndrome was defined based on International Diabetic Federation (IDF) criteria using waist circumference ( $\geq 94 \mathrm{~cm}$ (men), $\geq 80 \mathrm{~cm}$ (female), or two or more of the following. i) Fasting triglyceride $>150 \mathrm{~g} / \mathrm{dL}$ or specific treatment for this lipid abnormality. ii) HDL cholesterol $<40 \mathrm{mg} / \mathrm{dL}$ (men), $<50 \mathrm{mg} / \mathrm{dL}$ (women) or specific treatment for this lipid abnormality. iii) Blood pressure $>130 \mathrm{mmHg}$ (systolic), $>90 \mathrm{mmHg}$ (diastolic) or on anti-hypertensive treatment. iv)fasting plasma glucose $\geq 100$ $\mathrm{mg} / \mathrm{dL}$ or previously diagnosed diabetes mellitus. ${ }^{29}$

\section{Data management and statistical analysis}

The data was analyzed using Statistical Package for Social Sciences (SPSS), version 20.0 (IBM). The qualitative data were summarized as frequency and percentage while quantitative variables was summarized as means and standard deviations (SD). To investigate the statistical significance of the difference in continuous variables according to gender and the presence of metabolic syndrome or not, independent samples t-test was employed. For categorical variables, the Chi-square test for the compari- son of proportions was employed. Association between selected demographic, clinical characteristics and ECG findings was investigated at bivariate and multivariate levels with p-value $<0.05$.

\section{Results}

Four hundred and ninety-one participants comprising $48.3 \%$ women with mean age $53.72 \pm 15.2$ years who met the IDF criteria with complete ECG interpretations were analyzed. $44.2 \%$ (men $38.6 \%$; women $50.2 \%$ ) and $74 \%$ of the participants had MetS and ECG abnormalities respectively. As shown in Table 2 and 3, mean age, blood pressure parameters and waist circumference were comparable across gender. Compared to women, men had higher mean total cholesterol, creatinine, smoking, and alcohol use, and diabetes mellitus, QT prolongation, LVH plus or minus strain pattern, and ECG abnormalities in general. Women were heavier, had higher heart rate and proportions of MetS. Expectedly, participants with MetS compared with those without MetS had higher mean blood pressure, waist circumference, BMI, Triglyceride, fasting plasma glucose and smoking history. ECG findings were similar among the group.

Table 2. Characteristics of Study Participant by Gender

\begin{tabular}{|c|c|c|c|c|}
\hline Variables & $\begin{array}{l}\text { Total } \\
491(100 \%)\end{array}$ & $\begin{array}{l}\text { Male } \\
254 \\
(51.7 \%) \\
\end{array}$ & $\begin{array}{l}\text { Female } \\
237 \\
(48.3 \%) \\
\end{array}$ & $\begin{array}{l}\text { p- } \\
\text { Value }\end{array}$ \\
\hline Age (years) & $53.72 \pm 15.2$ & $54.37 \pm 15.5$ & $53.03 \pm 14.8$ & 0.328 \\
\hline Alcohol Use (\%) & $130(26.5)$ & $90(35.4)$ & $40(16.9)$ & $<0.0001$ \\
\hline Ex-Smoker (\%) & $114(23.2)$ & $82(32.3)$ & $32(13.5)$ & $<0.0001$ \\
\hline Current Smoker (\%) & $3(0.6)$ & $3(1.2)$ & $0(0.0)$ & \\
\hline Hip Circumference (cm) & $102.13 \pm 14.7$ & $99.68 \pm 13.6$ & $104.77 \pm 15.5$ & $<0.0001$ \\
\hline Waist Circumference (cm) & $93.09 \pm 14.8$ & $91.97 \pm 15.6$ & $94.30 \pm 13.8$ & 0.081 \\
\hline Body Mass Index $\left(\mathrm{Kg} / \mathrm{m}^{2}\right)$ & $27.34 \pm 5.8$ & $25.80 \pm 4.9$ & $28.98 \pm 6.1$ & $<0.0001$ \\
\hline Systolic Blood Pressure (mmHg) & $144.53 \pm 24.4$ & $145.33 \pm 24.0$ & $143.65 \pm 24.8$ & 0.449 \\
\hline Diastolic Blood Pressure (mmHg) & $89.13 \pm 16.5$ & $89.75 \pm 17.1$ & $88.44 \pm 15.8$ & 0.381 \\
\hline Fasting Blood Sugar (mg/dL) & $98.97 \pm 36.0$ & $102.02 \pm 41.1$ & $95.71 \pm 29.4$ & 0.069 \\
\hline Urea (mg/dL) & $28.30 \pm 17.6$ & $30.28 \pm 21.0$ & $26.26 \pm 12.8$ & 0.013 \\
\hline Creatinine (mg/dL) & $1.31 \pm 1.1$ & $1.25 \pm 0.8$ & $1.01 \pm 1.3$ & 0.014 \\
\hline Total Cholesterol (mg/dL) & $185.28 \pm 45.6$ & $180.14 \pm 46.4$ & $190.98 \pm 44.0$ & 0.011 \\
\hline Triglyceride (mg/dL) & $100.99 \pm 39.4$ & $102.90 \pm 45.1$ & $98.89 \pm 32.0$ & 0.275 \\
\hline $\begin{array}{l}\text { Low Density Lipoprotein Cholesterol } \\
(\mathrm{mg} / \mathrm{dL})\end{array}$ & $117.15 \pm 38.2$ & $114.07 \pm 37.8$ & $120.55 \pm 38.4$ & 0.069 \\
\hline High Density Lipoprotein (mg/dL) & $47.18 \pm 17.34$ & $44.94 \pm 16.0$ & $49.66 \pm 18.4$ & 0.003 \\
\hline \multicolumn{5}{|l|}{ ECG Changes } \\
\hline Heart Rate (beat/minute) & $84.01 \pm 18.3$ & $81.74 \pm 17.5$ & $86.38 \pm 19.0$ & 0.008 \\
\hline P Duration (ms) & $113.10 \pm 31.2$ & $111.94 \pm 30.1$ & $114.23 \pm 32.3$ & 0.445 \\
\hline QRS Duration (ms) & $113.64 \pm 30.8$ & $115.51 \pm 30.1$ & $111.71 \pm 31.4$ & 0.197 \\
\hline T Duration (ms) & $266.04 \pm 44.0$ & $266.41 \pm 42.2$ & $265.68 \pm 45.8$ & 0.890 \\
\hline PR Interval (ms) & $151.38 \pm 35.7$ & $150.10 \pm 33.7$ & $152.63 \pm 37.5$ & 0.471 \\
\hline QT Interval (ms) & $405.43 \pm 48.7$ & $405.32 \pm 46.0$ & $405.54 \pm 51.5$ & 0.963 \\
\hline QTe Interval (ms) & $\begin{array}{l}475.71 \pm \\
70.5\end{array}$ & $469.46 \pm 71.3$ & $482.13 \pm 69.3$ & 0.061 \\
\hline LVH (\%) & $153(32.7)$ & $92(38.2)$ & $61(26.9)$ & 0.009 \\
\hline LVH with Strain Pattern (\%) & $44(9.5)$ & $34(14.4)$ & $10(4.4)$ & $<0.0001$ \\
\hline Prolonged QT (\%) & $269(58.0)$ & $150(62.8)$ & $119(52.9)$ & 0.031 \\
\hline ECG Abnormalities (\%) & $356(74.0)$ & 199 (79.9) & $157(67.7)$ & 0.002 \\
\hline Diabetes Mellitus (\%) & $35(8.1)$ & $25(11.2)$ & $10(4.8)$ & 0.014 \\
\hline MetS (\%) & $217(44.2)$ & $98(38.6)$ & $119(50.2)$ & 0.010 \\
\hline
\end{tabular}


Table 3: Basic Demographic and Clinical Parameters versus Metabolic Syndrome

\begin{tabular}{|c|c|c|c|c|}
\hline Variables & $\begin{array}{l}\text { Total } \\
491(100 \%)\end{array}$ & $\begin{array}{l}\text { Non-Metabolic } \\
\text { Syndrome } \\
\text { (NMetS) } \\
274(55.8 \%) \\
\end{array}$ & $\begin{array}{l}\text { Metabolic } \\
\text { Syndrome } \\
\text { (MetS) } \\
217(44.2 \%) \\
\end{array}$ & p-Value \\
\hline Age (years) & $53.72 \pm 15.2$ & $53.06 \pm 16.7$ & $54.57 \pm 13.0$ & 0.262 \\
\hline Alcohol Use (\%) & $130(26.5)$ & $72(26.3)$ & $58(26.7)$ & 0.430 \\
\hline \multirow[t]{2}{*}{ Smoking Status } & Former Smoker (\%) & $114(23.2)$ & $76(27.7)$ & $38(17.5)$ \\
\hline & Current Smoker (\%) & $3(0.6)$ & $0(0.0)$ & $3(1.4)$ \\
\hline \multirow[t]{2}{*}{ Occupation } & Professional (\%) & $138(28.1)$ & $65(23.7)$ & $73(33.6)$ \\
\hline & Unskilled (\%) & $4(0.8)$ & $2(0.7)$ & $2(0.9)$ \\
\hline Hip Circumference (cm) & $102.13 \pm 14.7$ & $97.28 \pm 14.7$ & $108.26 \pm 12.3$ & $<0.0001$ \\
\hline Waist Circumference (cm) & $93.09 \pm 14.8$ & $87.24 \pm 13.8$ & $100.48 \pm 12.5$ & $<0.0001$ \\
\hline Hip Waist Ratio & $6.00 \pm 65.4$ & $9.12 \pm 88.1$ & $2.36 \pm 15.2$ & 0.418 \\
\hline Body Mass Index $\left(\mathrm{Kg} / \mathrm{m}^{2}\right)$ & $27.34 \pm 5.8$ & $25.31 \pm 5.3$ & $29.93 \pm 5.3$ & $<0.0001$ \\
\hline Waist Height Ratio & $0.58 \pm 0.3$ & $0.54 \pm 0.2$ & $0.63 \pm 0.4$ & $<0.0001$ \\
\hline Waist Hip Ratio & $0.93 \pm 0.4$ & $0.92 \pm 0.5$ & $0.93 \pm 0.1$ & 0.787 \\
\hline $\begin{array}{l}\text { Systolic Blood Pressure } \\
(\mathrm{mmHg})\end{array}$ & $144.53 \pm 24.4$ & $139.57 \pm 24.7$ & $150.69 \pm 22.5$ & $<0.0001$ \\
\hline $\begin{array}{l}\text { Diastolic Blood Pressure } \\
(\mathrm{mmHg})\end{array}$ & $89.13 \pm 16.5$ & $86.50 \pm 16.7$ & $92.39 \pm 15.6$ & $<0.0001$ \\
\hline Fasting Blood Glucose (mg/dL) & $98.97 \pm 36.0$ & $94.72 \pm 37.5$ & $103.94 \pm 33.7$ & 0.008 \\
\hline Urea $(\mathrm{mg} / \mathrm{dL})$ & $28.30 \pm 17.6$ & $28.92 \pm 21.2$ & $27.55 \pm 11.5$ & 0.401 \\
\hline Two Hours Fasting Blood & $121.06 \pm 55.0$ & $111.82 \pm 49.2$ & $130.55 \pm 59.0$ & 0.010 \\
\hline \multicolumn{5}{|l|}{ Sugar (mg/dL) } \\
\hline Creatinine (mg/dL) & $1.13 \pm 1.1$ & $1.121 \pm 0.8$ & $1.14 \pm 1.39$ & 0.821 \\
\hline Total Cholesterol (mg/dL) & $185.28 \pm 45.6$ & $184.85 \pm 46.5$ & $185.76 \pm 44.6$ & 0.831 \\
\hline Triglyceride (mg/dL) & $100.99 \pm 39.4$ & $93.54 \pm 35.0$ & $109.45 \pm 42.4$ & $<0.0001$ \\
\hline Low Density & $117.15 \pm 38.2$ & $115.49 \pm 38.5$ & $119.03 \pm 37.8$ & 0.320 \\
\hline \multicolumn{5}{|l|}{ Cholesterol (mg/dL) } \\
\hline $\begin{array}{l}\text { High Density Lipoprotein (mg/ } \\
\text { dL) }\end{array}$ & $47.18 \pm 17.3$ & $50.95 \pm 19.0$ & $42.94 \pm 14.1$ & $<0.0001$ \\
\hline
\end{tabular}

Tables 4, 5 and 6 depict gender differences in ECG changes and Components of MetS. In men IDF metabolic score was associated with conduction abnormalities $(p=0.039)$ and combined ECG abnormality $(p=0.042)$ which became more significant with the exclusion of QT prolongation $(\mathrm{p}=0.004)$. Also, IDF abdominal obesity was asso- ciated with QT prolongation $(\mathrm{p}=0.017)$, combined ECG abnormality $(\mathrm{p}=0.034)$, and HDLc with ECG abnormalities (0.037). No significant associations of components of metabolic syndrome with ECG abnormalities among women. Table 7 shows that male gender and HDLc were independent predictors of ECG Abnormalities.

Table 4: Comparisons of ECG abnormalities in metabolic syndrome

\begin{tabular}{lllll}
\hline Variables & Total & Non-Metabolic Syndrome & \multicolumn{2}{l}{ Metabolic Syndrome } \\
& $491(100 \%)$ & $274(55.8 \%)$ & $217(44.2 \%)$ & Value \\
\hline P Duration (ms) & $113.10 \pm 31.2$ & $111.77 \pm 33.5$ & $114.61 \pm 28.4$ & 0.356 \\
QRS Duration (ms) & $113.64 \pm 30.8$ & $115.04 \pm 32.6$ & $112.02 \pm 28.5$ & 0.306 \\
T Duration (ms) & $266.04 \pm 44.0$ & $267.63 \pm 47.6$ & $264.19 \pm 39.5$ & 0.515 \\
PR Interval (ms) & $151.38 \pm 35.7$ & $151.23 \pm 37.1$ & $151.55 \pm 34.1$ & 0.927 \\
QT Interval (ms) & $405.43 \pm 48.7$ & $407.18 \pm 51.3$ & $403.42 \pm 45.6$ & 0.423 \\
QTc Interval (ms) & $475.71 \pm 70.5$ & $479.64 \pm 79.9$ & $471.20 \pm 57.9$ & 0.203 \\
Left Atrial Enlargement (\%) & $47(9.6)$ & $28(10.2)$ & $19(8.8)$ & 0.787 \\
Bi-Atrial Enlargement (\%) & $11(2.2)$ & $7(2.6)$ & $4(1.8)$ & 0.789 \\
LVH (\%) & $153(31.2)$ & $93(33.9)$ & $60(27.6)$ & 0.324 \\
LV Strain Pattern (\%) & $44(9.0)$ & $26(9.5)$ & $18(8.3)$ & 0.885 \\
RV Hypertrophy (\%) & $12(2.4)$ & $9(3.3)$ & $3(1.4)$ & 0.385 \\
Prolonged QT interval (\%) & $269(58)$ & $146(57.6)$ & $123(58.6)$ & 0.85 \\
ECG Abnormalities (\%) & $356(74.0)$ & $200(74.3)$ & $156(73.6)$ & 0.917 \\
ECG Abnormalities \{Without & $255(53.6)$ & $152(57.4)$ & $103(48.8)$ & 0.065 \\
QT Abnormality\} (\%) & & & & \\
\hline
\end{tabular}


Table 5. Gender specific characteristics in metabolic syndrome

\begin{tabular}{|c|c|c|c|c|c|c|}
\hline \multirow[t]{2}{*}{ Variables } & Male & \multirow{2}{*}{$\begin{array}{l}\text { p-Value } \\
\text { Metabolic }\end{array}$} & \multirow[t]{2}{*}{ Female } & \multirow{2}{*}{$\begin{array}{l}\text { p-Value } \\
\text { Non- } \\
\text { Metabolic }\end{array}$} & \multirow[b]{2}{*}{ Metabolic } & \\
\hline & $\begin{array}{l}\text { Non- } \\
\text { Metabolic }\end{array}$ & & & & & \\
\hline Age (years) & $53.34 \pm 17.1$ & $56.03 \pm 12.6$ & 0.181 & $52.68 \pm 16.2$ & $53.36 \pm 13.2$ & 0.724 \\
\hline Body Mass Index $\left(\mathrm{kg} / \mathrm{m}^{2}\right)$ & $23.87 \pm 4.3$ & $28.93 \pm 4.2$ & $<0.0001$ & $27.22 \pm 5.9$ & $30.73 \pm 5.9$ & $<0.0001$ \\
\hline Hip Circumference $(\mathrm{cm})$ & $94.67 \pm 12.9$ & $107.65 \pm 10.7$ & $<0.0001$ & $100.74 \pm 16.3$ & $108.76 \pm 13.5$ & $<0.0001$ \\
\hline $\begin{array}{l}\text { Waist Circumference } \\
(\mathrm{cm})\end{array}$ & $84.81 \pm 12.5$ & $103.37 \pm 12.9$ & $<0.0001$ & $90.45 \pm 14.8$ & $98.11 \pm 11.9$ & $<0.0001$ \\
\hline $\begin{array}{l}\text { Systolic Blood Pressure } \\
(\mathrm{mmHg})\end{array}$ & $140.94 \pm 24.1$ & $152.29 \pm 22.3$ & $<0.0001$ & $137.73 \pm 25.5$ & $149.38 \pm 22.7$ & $<0.0001$ \\
\hline $\begin{array}{l}\text { Diastolic Blood Pressure } \\
(\mathrm{mmHg})\end{array}$ & $87.58 \pm 16.6$ & $93.19 \pm 17.4$ & 0.011 & $85.04 \pm 16.8$ & $91.73 \pm 14.1$ & 0.001 \\
\hline $\begin{array}{l}\text { Fasting Blood Sugar } \\
(\mathrm{mg} / \mathrm{dL})\end{array}$ & $98.03 \pm 46.0$ & $108.25 \pm 31.3$ & 0.070 & $90.07 \pm 19.6$ & $100.59 \pm 35.1$ & 0.010 \\
\hline Urea (mg/dL) & $30.71 \pm 25.2$ & $29.63 \pm 12.3$ & 0.700 & $26.68 \pm 14.7$ & $25.84 \pm 10.6$ & 0.620 \\
\hline Creatinine $(\mathrm{mg} / \mathrm{dL})$ & $1.28 \pm 0.9$ & $1.22 \pm 0.4$ & 0.546 & $0.93 \pm 0.4$ & $1.09 \pm 1.8$ & 0.362 \\
\hline $\begin{array}{l}\text { Total Cholesterol } \\
(\mathrm{mg} / \mathrm{dL})\end{array}$ & $176.23 \pm 45.8$ & $185.89 \pm 46.9$ & 0.112 & $197.15 \pm 44.8$ & $185.66 \pm 42.8$ & 0.054 \\
\hline Triglyceride (mg/dL) & $94.97 \pm 38.1$ & $114.56 \pm 51.9$ & 0.001 & $91.50 \pm 30.1$ & $105.20 \pm 32.3$ & 0.001 \\
\hline $\begin{array}{l}\text { Low Density Cholesterol } \\
(\mathrm{mg} / \mathrm{dL})\end{array}$ & $111.67 \pm 36.5$ & $117.59 \pm 39.5$ & 0.232 & $120.93 \pm 40.7$ & $120.23 \pm 36.5$ & 0.893 \\
\hline $\begin{array}{l}\text { High Density Lipoprotein } \\
(\mathrm{mg} / \mathrm{dL})\end{array}$ & $46.92 \pm 15.4$ & $42.05 \pm 16.7$ & 0.020 & $56.65 \pm 22.1$ & $43.67 \pm 11.6$ & $<0.0001$ \\
\hline $\begin{array}{l}\text { Heart Rate } \\
\text { (beats/minutes) }\end{array}$ & $83.26 \pm 17.7$ & $79.57 \pm 17.0$ & 0.120 & $86.35 \pm 18.9$ & $86.41 \pm 19.1$ & 0.982 \\
\hline P Duration (ms) & $112.01 \pm 33.7$ & $111.84 \pm 24.3$ & 0.968 & $111.49 \pm 33.4$ & $116.81 \pm 31.2$ & 0.233 \\
\hline QRS Duration (ms) & $113.85 \pm 32.2$ & $117.85 \pm 26.9$ & 0.332 & $116.53 \pm 33.2$ & $107.19 \pm 28.9$ & 0.029 \\
\hline T Duration (ms) & $267.62 \pm 42.9$ & $264.85 \pm 41.6$ & 0.704 & $267.64 \pm 2.5$ & $263.63 \pm 37.9$ & 0.602 \\
\hline PR Interval (ms) & $151.26 \pm 35.4$ & $148.52 \pm 31.4$ & 0.566 & $151.19 \pm 39.1$ & $153.99 \pm 36.1$ & 0.590 \\
\hline QT Interval (ms) & $404.36 \pm 49.8$ & $406.67 \pm 40.2$ & 0.713 & $410.67 \pm 53.1$ & $400.73 \pm 49.7$ & 0.158 \\
\hline QTc Interval (ms) & $471.51 \pm 78.6$ & $466.59 \pm 59.9$ & 0.614 & $489.71 \pm 80.7$ & $475.02 \pm 56.1$ & 0.121 \\
\hline
\end{tabular}


Table 6. Gender specific association between components of metabolic syndrome and ECG Abnormality

\begin{tabular}{|c|c|c|c|c|c|c|}
\hline \multirow[t]{2}{*}{ Variables } & Male & p-Value & Female & p-Value & & \\
\hline & $\begin{array}{l}\text { ECG- } \\
\text { Abnormality }\end{array}$ & $\begin{array}{l}\text { No ECG- } \\
\text { Abnormality }\end{array}$ & & $\begin{array}{l}\text { ECG- } \\
\text { Abnormality }\end{array}$ & $\begin{array}{l}\text { No ECG- } \\
\text { Abnormalit } \\
\text { y }\end{array}$ & \\
\hline $\begin{array}{l}\text { IDF- } \\
\text { Abdominal Obesity (\%) }\end{array}$ & $101(50.8)$ & $17(34.0)$ & 0.034 & $135(86.0)$ & $69(92.0)$ & 0.189 \\
\hline IDF-Blood Pressure (\%) & $170(81.3)$ & $39(78.0)$ & 0.173 & $122(78.2)$ & $55(75.3)$ & 0.630 \\
\hline IDF-Glucose (\%) & $64(36.8)$ & $12(26.1)$ & 0.175 & $37(26.8)$ & $15(28.8)$ & 0.460 \\
\hline IDF-Triglceride (\%) & $21(11.0)$ & $5(10.4)$ & 0.908 & $9(6.3)$ & $6(8.6)$ & 0.533 \\
\hline IDF-HDLc (\%) & $134(70.5)$ & $41(85.4)$ & 0.037 & $87(60.4)$ & $47(67.1)$ & 0.340 \\
\hline
\end{tabular}

Table 7: Determinants of ECG abnormalities in the study population

\begin{tabular}{llll}
\hline Variable & Odd ratio & $\mathbf{9 5 \%}$ Confidence Interval & P value \\
Age & 1.006 & $0.990-1.022$ & 0.460 \\
Male Gender & 1.904 & $1.126-3.220$ & 0.016 \\
IDF_Abdominal Obesity & 0.944 & $0.540-1.651$ & 0.839 \\
MS_Blood Glucose & 0.825 & $0.490-1.389$ & 0.469 \\
MS_Blood Pressure & 0.837 & $0.461-1.519$ & 0.558 \\
MS_High Density Lipoprotein & 1.843 & $1.068-3.180$ & 0.028 \\
MS_Triglyceride & 1.172 & $0.517-2.661$ & 0.704 \\
\hline
\end{tabular}

\section{Discussion}

In this study, approximately 4 out of 10 participants had MetS while three quarters had ECG abnormalities. Abnormal ECG findings were commoner in men with no differential association in people with MetS and those without MetS. However, a significant association existed between certain components of MetS and ECG abnormalities in men only.

The prevalence of $44.2 \%$ in this current study is almost twice the prevalence earlier reported by our team among the health workers. While women had excess MetS in both study cohorts, the gaps appear closing up among the gender, " $34.9 \%$ vs $2.4 \%$ among health workers as against $50.2 \%$ vs $38.6 \% . " 30$. Similarly, our finding of a high proportion of MetS was higher than others reported in Nigerian communities ${ }^{31}$. The rising trends may not be unconnected with equally increasing components such as obesity, hypertension, dyslipidemias and insulin resistance resulting from unhealthy lifestyles and epidemiologic transition ${ }^{32}$. May also be due to the fact that it was prevalence at a referral clinic.

Contrary to our findings, probably due to the influence of wealth and intake of atherogenic diets, Barrios and colleagues in Spain, ${ }^{33}$ Ford and colleagues ${ }^{34}$ in the United States, and Yassein and colleagues ${ }^{35}$ in Jordan reported higher prevalence rates of $52 \%, 62.9 \%$, and $52 \%$, respectively. With a high proportion of metabolic syndrome components (high BP, 80.7\%; central obesity, 67\%; low HDL, $68.7 \%$ and hyperglycemia, 30\%) in this cohort, MetS incidences will soon rival those of HICs. Apparently, our study population requires urgent care to prevent a possible escalation of stroke, heart failure, and Myocardial infarction associated with increasing prevalence of MetS. 
Abnormalities in resting electrocardiograph (ECG) have been strongly associated with cardiovascular morbidity and mortality. ${ }^{36}$ Its coexistence with MetS potentiates the rate of sudden cardiac deaths. ${ }^{37}$ In this study, we report a high prevalence of ECG abnormalities and MetS. There was no association between MetS and ECG abnormalities. However, central obesity and low HDL were significantly associated with ECG abnormalities in men. Similar to our findings, Ebong et.al in MESA study reported a high prevalence of ECG in Mets which were gender specific $^{38}$. However, they found associations between ECG abnormalities and MetS and its components; high blood pressure and triglyceride. The reasons for these discrepancies were not immediately obvious. Our finding, showing no association between ECG changes and MetS is similar to an earlier study in Nigeria ${ }^{17}$. Apart from the larger population in our study, gender-specific association between components of MetS and ECG abnormalities was not explored, which our study showed.

Similarly, a study in African Americans which share ancestry with blacks in sub-Saharan Africans showed high a proportion of ECG changes among their study population, so also among Caucasians ${ }^{16,39,40}$. Our study specifically showed a high prevalence of prolonged QTc, left ventricular hypertrophy (LVH) and LVH with strains abnormality similar to other studies ${ }^{16}$. This is not surprising since studies have shown high preponderance of LVH among blacks. ${ }^{41,42}$. Left ventricular hypertrophy is an independent risk factor for stroke. Prolongation of the QT and QTc intervals is caused by the heightened sympathetic activity characteristic of obesity that increases the reduced heart rate variability; all these elements have the potential to cause arrhythmia and may lead to cardio-embolic stroke.

Previous studies have established the role of uncontrolled elevated blood pressure and other traditional cardiovascular risk factors in structural myocardial changes which are interpreted from ECG recordings ${ }^{43}$. The aggregates of these risk factors which constitutes MetS are suspected to produce more abnormal ECG changes in people with MetS compared with those without MetS. Our study did not have significant changes between the two groups. However, certain components of MetS were significantly different among men only. High frequency of hypertension among the groups may account for the insignificant ECG findings.
The implication of our findings to clinical care is that people referred for cardiac care in our center who have high aggregates of cardiovascular risk factors should have an ECG done at presentation. This is because of the high prevalence of significant abnormalities which may be due to high prevalence of hypertension and dyslipidemia, the most important drivers of ECG changes, especially among blacks. ${ }^{17,44,45}$

\section{Strengths and limitation}

Our study has several strengths; one of such is establishing the burden of metabolic syndrome and abnormal ECG among the first timer at a cardiac clinic in Nigeria. Our findings have implications for early risk stratification of our patients for prompt management. However, the cross-sectional study design is a limitation, as the causal relationship cannot be established. Also the use of Sokolow Lyon criteria which is non sex-specific might have exaggerated our findings of LVH in men. In addition, the small sample size and hospital-based data collection limit the generalization of our findings to the general population.

\section{Conclusion}

MetS and abnormal ECG changes are common among the studied population. While women had a higher prevalence of MetS, men had more abnormal ECG findings with a significant association between abdominal obesity, dyslipidemia and ECG abnormalities in men only. Larger prospective study is required to support gender - specific cardiac care in this population.

\section{Disclosure}

None.

\section{Conflict of interest}

None declared.

\section{References}

1. Eckel RH, Grundy SM, Zimmet PZ. The metabolic syndrome. Lancet. 2005;365(9468):1415-28.

2. Misra A, Khurana L. Obesity and the Metabolic Syndrome in Developing Countries. The Journal of Clinical Endocrinology \& Metabolism. 93, Issue 11_(11 supplement_1, $1): \mathrm{s} 9-\mathrm{s} 30$.

3. Okafor CI. The metabolic syndrome in Africa: Current trends. Indian Journal of Endocrinology and Metabolism. 2012;16(1):56-66. 
4. Grundy SM. Metabolic syndrome pandemic. Arteriosclerosis, thrombosis, and vascular biology. 2008;28(4):629-36.

5. Oguoma VM, Nwose EU, Richards RS. Prevalence of cardio-metabolic syndrome in\&\#xa0;Nigeria: a systematic review. Public Health.129(5):413-23.

6. Dekker JM, Girman C, Rhodes T, Nijpels G, Stehouwer CDA, Bouter LM, et al. Metabolic Syndrome and 10-Year Cardiovascular Disease Risk in the Hoorn Study. Circulation. 2005;112(5):666-73.

7. Cuspidi C, Meani S, Valerio C, Sala C, Fusi V, Zanchetti A, et al. Age and Target Organ Damage in Essential Hypertension: Role of the Metabolic Syndrome. American Journal of Hypertension. 2007;20(3):296-303.

8. Gami AS, Witt BJ, Howard DE, Erwin PJ, Gami LA, Somers VK, et al. Metabolic syndrome and risk of incident cardiovascular events and death: a systematic review and meta-analysis of longitudinal studies. I Am Coll Cardiol. 2007;49(4):403-14.

9. Hess PL, Al-Khalidi HR, Friedman DJ, Mulder H, Kucharska-Newton A, Rosamond WR, et al. The metabolic syndrome and risk of sudden cardiac death: the atherosclerosis risk in communities study. Journal of the American Heart Association. 2017;6(8):e006103.

10. Cannon RO, Camici PG, Epstein SE. Pathophysiological dilemma of syndrome X. Circulation. 1992;85(3):88392.

11. Galassi A, Reynolds K, He J. Metabolic Syndrome and Risk of Cardiovascular Disease: A Meta-Analysis. The American Journal of Medicine. 119(10):812-9.

12. Schillaci G, Pirro M, Vaudo G, Gemelli F, Marchesi $\mathrm{S}$, Porcellati C, et al. Prognostic value of the metabolic syndrome in essential hypertension. Journal of the American College of Cardiology. 2004;43(10):1817-22.

13. Adeoye AM, Ogah OS, Ovbiagele B, Akinyemi R, Shidali V, Agyekum F, et al. Prevalence and Prognostic Features of ECG Abnormalities in Acute Stroke: Findings From the SIREN Study Among Africans. Global Heart. 2017;12(2):99-105.

14. Tanner RM, Baber U, Carson AP, Voeks J, Brown TM, Soliman EZ, et al. Association of the metabolic syndrome with atrial fibrillation among United States adults (from the REasons for Geographic and Racial Differences in Stroke [REGARDS] Study). Am J Cardiol. 2011;108(2):227-32.

15. Kim H-K, Kim C-H, Ko K-H, Park S-W, Park J-Y, Lee K-U. Variable Association between Components of the Metabolic Syndrome and Electrocardiographic Ab- normalities in Korean Adults. The Korean Journal of Internal Medicine. 2010;25(2):174-80.

16. Ebong IA, Bertoni AG, Soliman EZ, Guo M, Sibley CT, Chen YD, et al. Electrocardiographic abnormalities associated with the metabolic syndrome and its components: the multi-ethnic study of atherosclerosis. Metabolic Syndrome and Related Disorders. 2012;10(2):92-7.

17. Ajayi EA, Ajayi OA, Adeoti OA. Metabolic syndrome: prevalence and association with electrocardiographic abnormalities in Nigerian hypertensive patients. Metabolic Syndrome and Related Disorders. 2014;12(8):437-42.

18. Nabipour I, Amiri M, Imami SR, Jahfari SM, Shafeiae E, Nosrati A, et al. The metabolic syndrome and nonfatal ischemic heart disease; a population-based study. International Journal of Cardiology. 118(1):48-53.

19. Onat A, Ceyhan K, Başar Ö, Erer B, Toprak S, Sansoy V. Metabolic syndrome: major impact on coronary risk in a population with low cholesterol levels-a prospective and cross-sectional evaluation. Atherosclerosis. 2002;165(2):285-92.

20. Kaski JC, Collins P, Nihoyannopoulos P, Maseri A, Poole-Wilson PA, Rosano GMC. Cardiac syndrome X: Clinical characteristics and left ventricular function. Longterm follow-up study. 1995;25(4):807-14.

21. Sharma A, Kushner R. A proposed clinical staging system for obesity. Int J Obes. 2009;33(3):289-95.

22. Organization WH. Waist circumference and waist-hip ratio: Report of a WHO expert consultation, Geneva, 8-11 December 2008. 2011.

23. Warren TY, Wilcox S, Dowda M, Baruth M. Peer Reviewed: Independent Association of Waist Circumference With Hypertension and Diabetes in African American Women, South Carolina, 2007-2009. Prev Chronic Dis. 2012;9.

24. Prineas RJ, Crow RS, Zhang Z-M. The Minnesota code manual of electrocardiographic findings: Springer Science \& Business Media; 2009.

25. van Bree MD, Roos YB, van der Bilt IA, Wilde AA, Sprengers ME, de Gans K, et al. Prevalence and characterization of ECG abnormalities after intracerebral hemorrhage. Neurocritical Care. 2010;12(1):50-5.

26. Roman MJ, Kligfield P, Devereux RB, Niles NW, Hochreiter C, Halle A, et al. Geometric and functional correlates of electrocardiographic repolarization and voltage abnormalities in aortic regurgitation. Journal of the American College of Cardiology. 1987;9(3):500-8.

27. Okin PM, Devereux RB, Nieminen MS, Jern S, Oikar- 
inen L, Viitasalo M, et al. Relationship of the electrocardiographic strain pattern to left ventricular structure and function in hypertensive patients: the LIFE study. Journal of the American College of Cardiology. 2001;38(2):514-20.

28. Lepeschkin E, Surawicz B. The measurement of the QT interval of the electrocardiogram. Circulation. 1952;6(3):378-88.

29. Alberti KG, Zimmet P, Shaw J. Metabolic syndrome--a new world-wide definition. A Consensus Statement from the International Diabetes Federation. Diabetic medicine: A Journal of the British Diabetic Association. 2006;23(5):46980 .

30. Adeoye AM, Adewoye IA, Dairo DM, Adebiyi A, Lackland DT, Ogedegbe G, et al. Excess metabolic syndrome risks among women health workers compared with men. The Journal of Clinical Hypertension. 2015;17(11):8804.

31. Adediran O, Akintunde A, Edo A, Opadijo O, Araoye A. Impact of urbanization and gender on frequency of metabolic syndrome among native Abuja settlers in Nigeria. Journal of Cardiovascular Disease Research. 2012;3(3):1916.

32. Okafor CI. The metabolic syndrome in Africa: Current trends. Indian Journal of Endocrinology and Metabolism. 2012;16(1):56.

33. Barrios V, Escobar C, Calderón A, Llisterri JL, Alegría E, Muñiz J, et al. Prevalence of the Metabolic Syndrome in Patients With Hypertension Treated in General Practice in Spain: An Assessment of Blood Pressure and Low-Density Lipoprotein Cholesterol Control and Accuracy of Diagnosis. Journal of the cardiometabolic syndrome. 2007;2(1):9-15.

34. Ford ES, Giles WH, Dietz WH. Prevalence of the metabolic syndrome among US adults: findings from the third National Health and Nutrition Examination Survey. JAMA. 2002;287(3):356-9.

35. Yasein N, Ahmad M, Matrook F, Nasir L, Froelicher E. Metabolic syndrome in patients with hypertension attending a family practice clinic in Jordan/Le syndrome metabolique chez des patients hypertendus frequentant un service de medecine familiale en Jordanie. Eastern Mediterranean Health Journal. 2010;16(4):375.

36. De Bacquer D, De Backer G, Kornitzer M, Blackburn
H. Prognostic value of ECG findings for total, cardiovascular disease, and coronary heart disease death in men and women. Heart. 1998;80(6):570-7.

37. Elffers TW, de Mutsert R, Lamb HJ, Maan AC, Macfarlane PW, van Dijk KW, et al. Association of metabolic syndrome and electrocardiographic markers of subclinical cardiovascular disease. Diabetology \& Metabolic Syndrome. 2017;9(1):40.

38. Ebong IA, Bertoni AG, Soliman EZ, Guo M, Sibley CT, Chen Y-DI, et al. Electrocardiographic abnormalities associated with the metabolic syndrome and its components: the multi-ethnic study of atherosclerosis. Metabolic Syndrome and Related Disorders. 2012;10(2):92-7.

39. Jelavic MM, Babic Z, Pintaric H. Influence of the metabolic syndrome definitions on electrocardiographic abnormalities. Acta Medica. 2015;31:401.

40. Queen SR, Smulevitz B, Rentfro AR, Vatcheva KP, Kim H, McPherson DD, et al. Electrocardiographic abnormalities among Mexican Americans: correlations with diabetes, obesity, and the metabolic syndrome. World Journal of Cardiovascular Diseases. 2012;2(2):50.

41. Adeoye AM, Ovbiagele B, Kolo P, Appiah L, Aje A, Adebayo O, et al. Exploring Overlaps Between the Genomic and Environmental Determinants of LVH and Stroke: A Multicenter Study in West Africa. Global Heart. 2017;12(2):107-13. e5.

42. Salako B, Ogah O, Adebiyi A, Adedapo K, Bekibele $\mathrm{C}$, Oluleye $\mathrm{T}$, et al. Unexpectedly high prevalence of target-organ damage in newly diagnosed Nigerians with hypertension. Cardiovascular Journal of South Africa. 2007;18(2):77.

43. Adebayo AK, Oladapo OO, Adebiyi AA, Ogunleye OO, Ogah OS, Ojji DB, et al. Changes in left atrial dimension and function and left ventricular geometry in newly diagnosed untreated hypertensive subjects. Journal of Cardiovascular Medicine. 2008;9(6):561-9.

44. Okeahialam B, Alonge B, Puepet F, Pam S, Balogun M. Cardiovascular morbidity: a comparative study on diabetes mellitus and hypertension. South African Journal of Diabetes and Vascular Disease. 2012;9(2):55-60.

45. Bello- Sani F, Anumah F. Electrocardiographic abnormalities in persons with type 2 diabetes in Kaduna, Northern Nigeria. Internal Journal of Diabetes and Metabolism. 2009;17:99-103. 